\title{
BERNSTEIN AND JACKSON THEOREMS FOR THE HEISENBERG GROUP
}

\author{
SAVERIO GIULINI
}

(Received 4 October 1983)

Communicated by G. Brown

\begin{abstract}
We describe on the Heisenberg group $H_{n}$ a family of spaces $M(h, X)$ of functions which play a role analogous to the trigonometric polynomials in $T^{n}$ or the functions of exponential type in $\mathbf{R}^{n}$. In particular we prove that for the space $M(h, X)$, Jackson's theorem holds in the classical form while Bernstein's inequality hold in a modified form. We end the paper with a characterization of the functions of the Lipschitz space $\Lambda_{x}^{r}$ by the behavior of their best approximations by functions in the space $M(h, X)$.
\end{abstract}

1980 Mathematics subject classification (Amer. Math. Soc.): 41 A 17, 43 A 80.

\section{Introduction}

Let $G$ be $\mathbf{R}^{n}$ or the $n$-dimensional torus $T^{n}$ and $X=L^{p}(G)$ or $C_{0}(G)$. For every positive real number $h$, we consider the subspace of $X$ :

$$
M(h, X)=\{f \in X: \hat{f}(\lambda)=0 \text { if } \lambda \in \hat{G} \text { and }|\lambda|>h\} .
$$

More explicitly for $G=T^{n}, M(h, X)$ is the space of all trigonometric polynomials of degree equal or less than $h$, while, if $G=\mathbf{R}^{n}, M(h, X)$ consists of all entire functions of exponential type $h$ in $\mathbf{C}^{n}$ which, as functions of the variable $x \in \mathbf{R}^{n}$, lie in $X$.

It is well known that the following inequalities hold:

a) (Jackson's theorem) for every integer $N>0$, there exists a constant $C_{N}$ such that

$$
\operatorname{Inf}_{g \in M(h, X)}\|f-g\|_{X} \leqslant C_{N} \omega_{N}(1 / h, f, X)
$$

(C) 1985 Australian Mathematical Society $0263-6115 / 85 \$ A 2.00+0.00$ 
for every $f \in X$, where $\omega_{N}$ is the $N$-th modulus of smoothness;

b) (Bernstein's theorem) for every multiindex $I$ and every $f \in M(h, X)$

$$
\left\|D^{I} f\right\|_{X} \leqslant h^{\mid I}\|f\|_{X}
$$

Our goal is to find for $G$ the Heisenberg group $H_{n}$ a family of spaces $M(h, X)$ ( $h \in \mathbf{R}^{+}$) which satisfy conditions a) and b). For this purpose we consider the non-trivial representations $\pi_{\lambda}$ of the Heisenberg group $H_{n}$ and we suppose that $\pi_{\lambda}$ acts on the Bargmann space $\mathscr{H}_{\lambda}$. If $\hat{f}(\lambda)$ is the Fourier transform of a function $f \in X$, we denote by $\{\hat{f}(\lambda)\}_{\alpha, \beta}\left(\alpha, \beta \in \mathbf{N}^{n}\right)$ the matrix entries of $\hat{f}(\lambda)$ with respect to the canonical orthonormal basis in $\mathscr{H}_{\lambda}$.

We define the space $M(h, X)$ in the following way:

$$
M(h, X)=\left\{f \in X:\{\hat{f}(\lambda)\}_{\alpha, \beta}=0 \text { if }(2|\beta|+n)|\lambda|>h^{2}\right\} .
$$

We prove that for these spaces Jackson's theorem holds in the classical form, while Bernstein's inequality holds in a modified form, with a constant greater than one. Finally we give a characterization of the functions of the Lipschitz spaces $\Lambda_{X}^{r}$ by the behavior of their best approximations by functions of the classes $M(h, X)$.

\section{Notation}

The Heisenberg group $H_{n}$ is the Lie group whose underlying manifold is $\mathbf{R} \times \mathbf{C}^{n}$ and whose composition law is given by

$$
(t, z) \cdot\left(t^{\prime}, z^{\prime}\right)=\left(t+t^{\prime}+2 \operatorname{Im} z \cdot \bar{z}^{\prime}, z+z^{\prime}\right)
$$

where $t, t^{\prime} \in \mathbf{R}, z=\left(z_{1}, \ldots, z_{n}\right), z^{\prime}=\left(z_{1}^{\prime}, \ldots, z_{n}^{\prime}\right) \in \mathbf{C}^{n}$ and $z \cdot z^{\prime}=\sum_{j=1}^{n} z_{j} \bar{z}_{j}^{\prime}$. The complexified Heisenberg Lie algebra $\left(\mathfrak{h}_{n}\right)_{C}$ is generated by the left-invariant vector fields

$$
T=\frac{\partial}{\partial t}, \quad Z_{j}=\frac{\partial}{\partial z_{j}}+i \bar{z}_{j} \frac{\partial}{\partial t}, \quad \bar{Z}_{j}=\frac{\partial}{\partial \bar{z}_{j}}-i z_{j} \frac{\partial}{\partial t} \quad(j=1, \ldots, n) .
$$

We denote by $V$ the vector space spanned by $Z_{j}, \bar{Z}_{j}(j=1, \ldots, n)$. Since the only non-trivial commutation rule is $\left[Z_{j}, \bar{Z}_{j}\right]=-2 i T, V$ generates $\left(\mathfrak{h}_{n}\right)_{\mathbf{C}}$ as an algebra (that is $H_{n}$ is stratified). The natural dilations on $\left(\mathfrak{h}_{n}\right)_{C}$ are given by

$$
\delta_{\varepsilon}(T+Z)=\varepsilon^{2} T+\varepsilon Z \quad(Z \in V, \varepsilon>0) .
$$

We shall denote also by $\delta_{\varepsilon}$ the corresponding dilations on $H_{n}$ :

$$
\delta_{\varepsilon}(t, z)=\left(\varepsilon^{2} t, \varepsilon z\right) \quad(t, z) \in H_{n} .
$$

We define on $H_{n}$ a homogeneous norm

$$
\rho(t, z)=\left(t^{2}+|z|^{4}\right)^{1 / 4}
$$


If $D$ is an invariant differential operator on $H_{n}$, we say that $D$ is homogeneous of degree $N$ if

$$
D\left(f \circ \delta_{\varepsilon}\right)=\varepsilon^{N}\left(D f \circ \delta_{\varepsilon}\right) .
$$

In particular $N=1$ if and only if $D \in V$.

The Haar measure on $H_{n}$ is Lebesgue measure on $\mathbf{R} \times \mathbf{C}^{n}$. Let $X$ denote either $L^{p}\left(H_{n}\right)$ or $C_{0}\left(H_{n}\right)$. The subspace $Y \subset X$ is defined as the space of all infinitely differentiable $f$ such that $D f \in X$ for every invariant differential operator $D$. We set

$$
f_{(\varepsilon)}=\varepsilon^{-2(n+1)} f \circ \delta_{1 / \varepsilon} \quad(\varepsilon>0) .
$$

Clearly, if $f \in L^{1}\left(H_{n}\right)$ one has

$$
\int_{H_{n}} f_{(\varepsilon)}=\int_{H_{n}} f
$$

and, if $f \in Y$ and $D$ is an invariant differential operator homogeneous of degree $N$,

$$
D f_{(\varepsilon)}=\varepsilon^{-N}(D f)_{(\varepsilon)} .
$$

In defining Fourier transforms for $H_{n}$ (see [4]) we are concerned only with the infinite-dimensional irreducible unitary representations of $H_{n}$. These representations can be considered as acting on the Bargmann space $\mathscr{H}_{\lambda}(\lambda>0)$ which consist of all holomorphic functions $F$ in $\mathbf{C}^{n}$ such that

$$
\|F\|^{2}=(2 \lambda / \pi)^{n} \int_{\mathbf{C}^{n}}|F(w)|^{2} \exp \left(-2 \lambda|w|^{2}\right) d w<+\infty .
$$

The monomials

$$
F_{\alpha, \lambda}(w)=(\sqrt{2 \lambda} w)^{\alpha} / \sqrt{\alpha !}, \quad \alpha \in \mathbf{N}^{n},
$$

form an orthonormal basis for the Hilbert space $\mathscr{H}_{\lambda}$. For $\lambda \in \mathbf{R}^{*}=\mathbf{R}-\{0\}$ the representations $\pi_{\lambda}$ on $\mathscr{H}_{|\lambda|}$ are given by

$$
\left(\pi_{\lambda}(t, z) F\right)(w)=F(w-\bar{z}) \exp \left(i \lambda t+2 \lambda\left(w \cdot z-|z|^{2} / 2\right)\right)
$$

if $\lambda>0$, and $\pi_{\lambda}(t, z)=\pi_{|\lambda|}(-t,-\bar{z})$ if $\lambda<0$; these exhaust all non-trivial irreducible unitary representations of $H_{n}$. The Fourier transform of a $L^{1}$-function $f$ is the operator valued function

$$
\hat{f}(\lambda)=\int_{H_{n}} f(u) \pi_{\lambda}(u) d u
$$

Let $\mathscr{F}_{\lambda}$ be the linear span of $\left\{F_{\alpha, \lambda}\right\}_{\alpha \in \mathbf{N}^{n}}$ and let $\mathscr{R}$ be the set of all families $S=\{S(\lambda)\}_{\lambda \in \mathbf{R}^{*}}$ of linear operators $S(\lambda): \mathscr{F}_{\lambda} \rightarrow \mathscr{H}_{\lambda}$. The matrix of $S(\lambda)$ is defined

$$
\{S(\lambda)\}_{\alpha, \beta}=\left(S(\lambda) F_{\alpha, \lambda}, F_{\beta, \lambda}\right)_{\mathscr{H}_{\lambda}}
$$


If $f \in S\left(H_{n}\right)$, the Schwartz space, the Plancherel formula

$$
\|f\|_{2}^{2}=c_{n} \int_{\mathbf{R}^{*}}\|\hat{f}(\lambda)\|_{H S}^{2}|\lambda|^{n} d \lambda
$$

holds, where $\|\cdot\|_{H S}$ is the Hilbert Schmidt norm and $c_{n}=2^{n-1} / \pi^{n+1}$. Then we can extend the Fourier transformation to an isometry from $L^{2}\left(H_{n}\right)$ onto the Hilbert space $\mathscr{L}^{2}$, where

$$
\begin{aligned}
& \mathscr{L}^{2}=\left\{S=\{S(\lambda)\} \in \mathscr{R}:\|S(\lambda)\|_{H S}<+\infty \text { for almost all } \lambda\right. \\
& \text { and } \left.\int_{\mathbf{R}^{*}}\|S(\lambda)\|_{H S}^{n}|\lambda|^{n} d \lambda<+\infty\right\} .
\end{aligned}
$$

More generally let $S^{\prime}\left(H_{n}\right)$ be the conjugate dual of $S\left(H_{n}\right)$; we define (see [4])

$$
S\left(h_{n}\right)=\left\{S \in \mathscr{R}: S=\hat{f} \text { for some } f \in S\left(H_{n}\right)\right\},
$$

and we topologize it to be homeomorphic to $S\left(H_{n}\right)$. Let $S^{\prime}\left(h_{n}\right)$ be the conjugate dual of $S\left(h_{n}\right)$. By polarization of the Plancherel formula we can extend the Fourier transformation to an isomorphism between $S^{\prime}\left(H_{n}\right)$ and $S^{\prime}\left(h_{n}\right)$ which we also denote by ${ }^{\wedge}$. If $\{R(\lambda)\} \in \mathscr{R}$, we shall say that $\{R(\lambda)\} \in S^{\prime}\left(h_{n}\right)$ if the map

$$
S \rightarrow \int_{\mathbf{R}^{*}} \sum_{\alpha \in \mathbf{N}^{n}}\left|\left(R(\lambda) F_{\alpha, \lambda}, S(\lambda) F_{\alpha, \lambda}\right)_{\mathscr{H}_{\lambda}}\right||\lambda|^{n} d \lambda
$$

is defined and continuous from $S\left(h_{n}\right)$ to $\mathrm{C}$ (see [5], Chapter 2).

We observe that, if $f, g \in S^{\prime}\left(H_{n}\right)$,

$$
\begin{aligned}
& \widehat{Z_{j} f}(\lambda) F_{\alpha, \lambda}=-\left(2|\lambda|\left(\alpha_{j}+1\right)\right)^{1 / 2} \hat{f}(\lambda) F_{\alpha+e_{j}, \lambda}, \\
& \widehat{\overline{Z_{j}} f}(\lambda) F_{\alpha, \lambda}=\left(2|\lambda| \alpha_{j}\right)^{1 / 2} \hat{f}(\lambda) F_{\alpha-e_{j}, \lambda},
\end{aligned}
$$

(where $\left\{e_{j}\right\}_{j=1, \ldots, n}$ is the canonical basis for $\mathbf{R}^{n}$, if $\lambda<0$ we must reverse the right sides) and

$$
D(f * g)=f * D g
$$

(where $D$ is any left invariant differential operator).

\section{Moduli of smoothness in $H_{n}$}

Moduli of smoothness in $H_{n}$ were studied by I. R. Inglis [6]. The results of this Section are analogous to the results obtained by P. M. Soardi [7] in $\mathbf{R}^{n}$ in the non-isotropic case. 
For every integer $N$ and every $0 \leqslant \theta \leqslant 1$ we define

$$
\Delta_{u, \theta}^{N} f\left(u^{\prime}\right)=\sum_{j=0}^{N}(-1)^{N+j}\left(\begin{array}{c}
N \\
j
\end{array}\right) f\left(u^{\prime} \cdot \delta_{j+\theta} u\right), \quad u, u^{\prime} \in H_{n}, f \in X .
$$

We set $\Delta_{u, 0}^{N} f=\Delta_{u}^{N} f$ and we remark that $\Delta_{u}^{N+1} f=\Delta_{u, 1}^{N} f-\Delta_{u}^{N} f$.

Definition 1. For every $h \in \mathbf{R}^{+}$and $N \in \mathbf{N}$, the function

$$
\omega_{N}(h, f, X)=\operatorname{Sup}_{\rho(u) \leqslant h}\left\|\Delta_{u}^{N} f\right\|_{X}
$$

is called the $N$-th modulus of smoothness.

For ease of notation we shall write $\omega_{N}(h, f)$ and $\|\cdot\|$ instead of $\omega_{N}(h, f, X)$ and $\|\cdot\|_{X}$. Since $H_{n}$ is stratified the space of left-invariant operators which are homogeneous of degree $N$ is exactly the linear span of the monomials $X_{1} X_{2} \cdots$ $X_{N}$, where $X_{i}=Z_{j}$ or $\bar{Z}_{k}(i=1, \ldots, N ; j, k=1, \ldots, n)$. We denote by $V_{N}$ the set of all differential monomials in $Z_{j}$ and $\bar{Z}_{k}(j, k=1, \ldots, n)$. Obviously $V=V_{1}$.

Lemma 1. Suppose $D \in V_{N}$. There exists a constant $C=C(D)$ such that for every $h>0$ and $f \in X$

$$
\|f-g\|+h^{N}\|D g\| \leqslant C \omega_{N}(h, f)
$$

for some $g \in Y$.

Proof. We choose $\phi \in S\left(H_{n}\right)$ such that $\int_{H_{n}} \phi=1$ and $\operatorname{supp} \phi \subseteq\left\{u \in H_{n}\right.$ : $\left.\rho(u) \leqslant 1 / N^{2}\right\}$. We define

$$
\begin{aligned}
& P=\frac{1}{N !} \sum_{j=1}^{N}(-1)^{N+j}\left(\begin{array}{l}
N \\
j
\end{array}\right) j^{N} \phi_{(j)}, \\
& Q=\sum_{j=1}^{N}(-1)^{N+j}\left(\begin{array}{l}
N \\
j
\end{array}\right) P_{(j)}
\end{aligned}
$$

(for the definition of $\phi_{(j)}$ and $P_{(j)}$ see (2)). Obviously $\int_{H_{n}} P=1, \int_{H_{n}} Q=(-1)^{N+1}$ and $\operatorname{supp} Q \subseteq\left\{u \in H_{n}: \rho(u) \leqslant 1\right\}$. We define $g=(-1)^{N+1} f * Q_{(h)}$. By changing variables one sees that

$$
\begin{aligned}
g(u)-f(u)= & \int_{\rho(v) \leqslant h} h^{-2(n+1)} P\left(\delta_{1 / h} v^{-1}\right) \\
& \cdot \sum_{j=0}^{N}(-1)^{N+j}\left(\begin{array}{c}
N \\
j
\end{array}\right) f\left(u \cdot \delta_{j} v\right) d v .
\end{aligned}
$$


Then

$$
\begin{aligned}
\|f-g\| & \leqslant \int_{\rho(v) \leqslant h}\left|P_{(h)}\left(v^{-1}\right)\right|\left\|\Delta_{v}^{N} f\right\| d v \\
& \leqslant\|P\|_{1} \omega_{N}(h, f) .
\end{aligned}
$$

Furthermore

$$
Q_{(h)}=\left(\frac{1}{N !}\right) \sum_{r, s=1}^{N}(-1)^{r+s}\left(\begin{array}{c}
N \\
r
\end{array}\right)\left(\begin{array}{c}
N \\
s
\end{array}\right) r^{N} \phi_{(h r s)}
$$

and by (1),

$$
D Q_{(h)}=\frac{h^{-N}}{N !} \sum_{r, s=1}^{N}(-1)^{r+s}\left(\begin{array}{c}
N \\
r
\end{array}\right)\left(\begin{array}{l}
N \\
s
\end{array}\right)\left(D \phi_{(s)}\right)_{(h r)} .
$$

A change of variables shows that

$$
\begin{aligned}
f * D Q_{(h)}= & \left(h^{-N} / N !\right) \sum_{s=1}^{N}(-1)^{s}\left(\begin{array}{c}
N \\
s
\end{array}\right) \int_{\rho(v) \leqslant h_{r=1}} \sum_{r=1}^{N}(-1)^{r}\left(\begin{array}{c}
N \\
r
\end{array}\right) \\
& \cdot f\left(u \cdot \delta_{r} v\right)\left(D \phi_{(s)}\right)_{(h)}\left(v^{-1}\right) d v .
\end{aligned}
$$

Since $\int_{\rho(v) \leqslant h}\left(D \phi_{(s)}\right)_{(h)}\left(v^{-1}\right) d v=0$, we have

$$
\begin{aligned}
h^{N}\|D g\| & =h^{N}\left\|f D Q_{(h)}\right\| \\
& \leqslant \frac{1}{N !} \sum_{s=1}^{N}\left(\begin{array}{c}
N \\
s
\end{array}\right) \int_{\rho(v) \leqslant h}\left|\left(D \phi_{(s)}\right)_{(h)}\left(v^{-1}\right)\right|\left\|\Delta_{v}^{N} f\right\| d v \\
& \leqslant \frac{1}{N !} \sum_{s=1}^{N}\left(\begin{array}{c}
N \\
s
\end{array}\right)\left\|D \phi_{(s)}\right\|_{1} \omega_{N}(h, f) .
\end{aligned}
$$

Lemma 2. Let $N$ be a positive integer and $0 \leqslant \theta \leqslant 1$. There exists a positive constant $C$, not depending on $\theta$, such that for every $f \in X, g \in Y$ and $h>0$

$$
\left\|\Delta_{u, \theta}^{N} f\right\| \leqslant C\left(\|f-g\|+\rho(u)^{N} \sum_{D \in V_{N}}\|D g\|\right) .
$$

Proof. Since

$$
\left\|\Delta_{u, \theta}^{N} f\right\| \leqslant\left\|\Delta_{u, \theta}^{N}(f-g)\right\|+\left\|\Delta_{u, \theta}^{N} g\right\| \leqslant 2^{N}\|f-g\|+\left\|\Delta_{u, \theta}^{N} g\right\|,
$$

we have to evaluate $\Delta_{u, \theta}^{N} g$ when $g \in Y$. Suppose $v=\left(0, z_{1}, \ldots, z_{n}\right) \in \exp V$; we observe that

$$
\left.\frac{d^{m}}{d s^{m}}\left(g\left(u^{\prime \prime} \cdot \delta_{s} v\right)\right)\right|_{s=j}=\left[\left(\sum_{k=1}^{n}\left(z_{k} Z_{k}+\bar{z}_{k} \bar{Z}_{k}\right)\right)^{m} g\right]\left(u^{\prime \prime} \cdot \delta_{j} v\right) .
$$


We set

$$
\sum_{k=1}^{n}\left(z_{k} Z_{k}+\bar{z}_{k} \bar{Z}_{k}\right)=E(v)
$$

Moreover there exist a constant $C^{\prime}>0$ and an integer $M$ such that any $u \in H_{n}$ can be expressed as

$$
\begin{aligned}
& u=v_{1} \cdot \ldots \cdot v_{M} \text { where } v_{i}=\left(0, w_{i}\right) \in \exp V \text { and } \rho\left(v_{i}\right) \leqslant \\
& C^{\prime} \rho(u)(i=1, \ldots, M \text {; see [3], Lemma 1.40). }
\end{aligned}
$$

Therefore an application of Taylor's theorem to the function $s \rightarrow g\left(u^{\prime \prime} \cdot \delta_{s} v_{i}\right)$ $\left(u^{\prime \prime} \in H ; i=1, \ldots, M\right)$ yields:

(8)

$$
\begin{aligned}
& g\left(u^{\prime} \cdot \delta_{j+\theta} u\right)-g\left(u^{\prime}\right)= \sum_{i=1}^{M} g\left(u^{\prime} \cdot \delta_{j+\theta}\left(v_{1} \cdot \ldots \cdot v_{i-1}\right) \cdot \delta_{j+\theta} v_{i}\right) \\
&-g\left(u^{\prime} \cdot \delta_{j+\theta}\left(v_{1} \cdot \ldots \cdot v_{i-1}\right)\right) \\
&= \sum_{i=1}^{M} \sum_{m=1}^{N-1} \frac{(j+\theta)^{m}}{m !}\left[E\left(v_{i}\right)^{m} g\right]\left(u^{\prime} \cdot \delta_{j+\theta}\left(v_{1} \cdot \ldots \cdot v_{i-1}\right)\right) \\
&+\frac{(j+\theta)^{N}}{(N-1) !} \int_{0}^{1}(1-s)^{N-1} \\
& \cdot\left[E\left(v_{i}\right)^{N} g\right]\left(u^{\prime} \cdot \delta_{j+\theta}\left(v_{1} \cdot \ldots \cdot v_{i-1} \cdot \delta_{s} v_{i}\right)\right) d s .
\end{aligned}
$$

By the inequality $\rho(u \cdot v) \leqslant \rho(u)+\rho(v)$ (see [2]), we have

$$
\rho\left(v_{1} \cdots v_{i-1} \delta_{s} v_{i}\right) \leqslant \sum_{k=1}^{i} \rho\left(v_{k}\right) \leqslant C^{\prime} M \rho(u) \quad(i=1, \ldots, M) .
$$

Furthermore

$$
\begin{aligned}
{\left[E\left(v_{i}\right)^{m} g\right]\left(u^{\prime} \cdot \delta_{j+\theta}\left(v_{1} \cdot \ldots \cdot v_{i-1}\right)\right)=} & \sum_{k=0}^{N-m} \frac{(j+\theta)^{k}}{k !}\left[E\left(v_{i-1}\right)^{k}\left(E\left(v_{i}\right)^{m} g\right)\right] \\
& \cdot\left(u^{\prime} \cdot \delta_{j+\theta}\left(v_{1} \cdot \ldots \cdot v_{i-2}\right)\right) \\
& \text { +remainder term. }
\end{aligned}
$$

Repeating this process we obtain

$$
\begin{aligned}
g\left(u^{\prime} \cdot \delta_{j+\theta} u\right)= & \sum_{s_{M}=0}^{N-1}(j+\theta)^{s_{M}} \sum_{s_{M-1}=0}^{s_{M}} \cdots \sum_{s_{1}=0}^{s_{2}}\left(s_{1} ! \prod_{k=2}^{M}\left(\left(s_{k}-s_{k-1}\right) !\right)\right)^{-1} \\
& \cdot\left[E\left(v_{1}\right)^{s_{1}} E\left(v_{2}\right)^{s_{2}-s_{1}} \cdots E\left(v_{M}\right)^{s_{M}-s_{M-1}} g\right]\left(u^{\prime}\right) \\
& + \text { remainder terms } \\
= & \sum_{s_{M}=0}^{N-1}(j+\theta)^{s_{M}} Q_{s_{M}}\left(u, u^{\prime}\right)+R\left(u, u^{\prime}, j+\theta\right)
\end{aligned}
$$


where $R\left(u, u^{\prime}, j+\theta\right)$ consists of all remainder terms, hence it can be written as a sum of terms like

$$
(j+\theta)^{N} \int_{0}^{1}(1-s)^{N-1-k} w^{\alpha} \bar{w}^{\beta}[D g]\left(u^{\prime} \cdot u(s)\right) d s
$$

where $D \in V_{N}$ and $0 \leqslant k \leqslant N-1 ; w=\left(w_{1}, \ldots, w_{n}\right) \in C^{n M}$ with $v_{i}=\left(0, w_{i}\right)$ and $\left|w_{i}\right| \leqslant C^{\prime} \rho(u)$ by (8); $\alpha, \beta \in N^{n M}$ and $|\alpha|+|\beta|=N ; \rho(u(s)) \leqslant(j+\theta) C^{\prime} M \rho(u)$ by (9). Now

$$
\begin{aligned}
\Delta_{u, \theta}^{N} g\left(u^{\prime}\right)= & \sum_{j=0}^{N}(-1)^{N+j}\left(\begin{array}{c}
N \\
j
\end{array}\right) g\left(u^{\prime} \cdot \delta_{j+\theta} u\right) \\
= & \sum_{s_{M}=0}^{N-1} \sum_{j=0}^{N}(j+\theta)^{s_{M}}(-1)^{N+j}\left(\begin{array}{c}
N \\
j
\end{array}\right) Q_{s_{M}}\left(u, u^{\prime}\right) \\
& +\sum_{j=0}^{N}(-1)^{N+j}\left(\begin{array}{c}
N \\
j
\end{array}\right) R\left(u, u^{\prime}, j+\theta\right) .
\end{aligned}
$$

Since $\sum_{j=0}^{N} j^{k}(-1)^{j}\left(\begin{array}{c}N \\ j\end{array}\right)=0$ if $k<N$, the first term in the previous sum is 0 ; it follows from (10) that

$$
\|R(u, \cdot, j+\theta)\| \leqslant C^{\prime \prime}(j+\theta)^{N} \rho(u)^{N} \sum_{D \in V_{N}}\|D g\| .
$$

Because $0 \leqslant \theta \leqslant 1$, finally we obtain

$$
\left\|\Delta_{u, \theta}^{N} g\right\| \leqslant C^{\prime \prime \prime} \rho(u)^{N} \sum_{D \in V_{N}}\|D g\|,
$$

where $C^{\prime \prime \prime}$ depends only on $N$.

We can summarize Lemmas 1 and 2 in the following statement: let $N$ be a positive integer; there exist two constants $C_{1}$ and $C_{2}$ such that for every $f \in X$ and $h>0$

$$
C_{1} \omega_{N}(h, f) \leqslant \inf _{g \in Y}\left(\|f-g\|+h^{N} \sum_{D \in V_{N}}\|D g\|\right) \leqslant C_{2} \omega_{N}(h, f) .
$$

The second member of (12) is the analogue in $H_{n}$ of the classical Peetre $K$-functional (see also [7]).

Corollary 1. Let $\varepsilon>0$, then

$$
\omega_{N}(\varepsilon h, f) \leqslant C(N)\left(1+\varepsilon^{N}\right) \omega_{N}(h, f) .
$$

Proof. Obvious from (12). 
COROLlaRY 2. Let $N$ be a positive integer. There exists a constant $C$ such that

$$
\left\|\Delta_{u, \theta}^{N} f\right\| \leqslant C \omega_{N}(\rho(u), f)
$$

for every $f \in X, u \in H_{n}$ and $0 \leqslant \theta \leqslant 1$.

LeMmA 3. Let $K$ and $k$ be two positive integers such that $K \geqslant k$. We suppose $\mathrm{g} \in X$ and $D \mathrm{~g} \in X$ for every $\mathrm{D} \in V_{i}(i \leqslant k)$. Then

$$
\left\|\Delta_{u}^{K} g\right\| \leqslant C(k) \rho(u)^{k} \sum_{D \in V_{k}} \omega_{K-k}(\rho(u), D g) \text {. }
$$

Proof. If we set $\theta=0$ and $N=1$ in formula ( 8 ), we get

$$
\begin{aligned}
\Delta_{u}^{K} g\left(u^{\prime}\right)= & \sum_{j=1}^{K}(-1)^{K+j}\left(g\left(u^{\prime} \cdot \delta_{j} u\right)-g\left(u^{\prime}\right)\right) \\
= & \sum_{i=1}^{M} \sum_{j=1}^{K} j(-1)^{K+j}\left(\begin{array}{c}
K \\
j
\end{array}\right) \int_{0}^{1}\left[E\left(v_{i}\right) g\right]\left(u^{\prime} \cdot \delta_{j}\left(v_{1} \cdot \ldots \cdot v_{i-1} \cdot \delta_{s} v_{i}\right)\right) d s \\
= & \sum_{i=1}^{M} \sum_{j=0}^{K-1}(-1)^{j+K-1}\left(\begin{array}{c}
K-1 \\
j
\end{array}\right) \\
& \cdot \int_{0}^{1}\left[E\left(v_{i}\right) g\right]\left(u^{\prime} \cdot \delta_{j+1}\left(v_{1} \cdot \ldots \cdot v_{i-1} \cdot \delta_{s} v_{i}\right)\right) d s .
\end{aligned}
$$

Now

$$
\begin{aligned}
& \left\|\Delta_{v_{1} \cdots v_{i-1} \cdot \delta_{s} v_{i}, 1}^{K-1} E\left(v_{i}\right) g\right\| \leqslant C^{\prime} \rho(u) \sum_{D \in V}\left\|\Delta_{v_{1} \cdot \ldots \cdot v_{i-1} \cdot \delta_{s} v_{i}, 1}^{K-1} D g\right\| \quad(\text { by }(6) \text { and (7)) } \\
& \leqslant C^{\prime \prime} \rho(u) \sum_{D \in V} \omega_{K-1}(C M \rho(u), D g) \quad(\text { by (9) and Corollary 2) } \\
& \leqslant C^{\prime \prime \prime} \rho(u) \sum_{D \in V} \omega_{K-1}(\rho(u), D g) \quad \text { (by Corollary 1). }
\end{aligned}
$$

Therefore

$$
\begin{aligned}
\left\|\Delta_{u}^{K} g\right\| & \leqslant \sum_{i=1}^{M} \int_{0}^{1}\left\|\Delta_{v_{1} \cdot \ldots \cdot v_{i-1} \cdot \delta_{s} u_{i}}^{K-1} E\left(v_{i}\right) g\right\| d s \\
& \leqslant M C^{\prime \prime \prime} \rho(u) \sum_{D \in V} \omega_{K-1}(\rho(u), D g) .
\end{aligned}
$$

Repeating this process, we obtain the thesis. 


\section{Jackson and Bernstein theorems}

Before proving the main theorems we observe that given a radial Schwartz function $f$ in $H_{n}$ (in the sense that $f(t, z)=f(t,|z|)$ ) we have $\{\hat{f}(\lambda)\}_{\alpha, \beta} \equiv 0$ if $\alpha \neq \beta$ and

$$
\{\hat{f}(\lambda)\}_{\alpha, \alpha}=\int_{\mathbf{R} \times \mathbf{C}^{n}} f(t, z) e^{i \lambda t} l_{\alpha}^{0}\left(2|\lambda||z|^{2}\right) d t d z
$$

where $l_{\alpha}^{0}$ is the Laguerre function of type 0 and degree $\alpha$ [4].

Definition 2. Let $h>0$. We denote by $M(h, X)$ the class of all functions $f \in X$ such that

$$
\{\hat{f}(\lambda)\}_{\alpha, \beta}=0 \quad \text { if }(2|\beta|+n)|\lambda|>h^{2} .
$$

THeOrem 1. Let $N$ be a positive integer. For every $f \in X$ and every $h>0$ there exists a function $g_{h} \in M(h, X)$ such that

$$
\left\|f-g_{h}\right\| \leqslant C(N) \omega_{N}(1 / h, f),
$$

where $C(N)$ is a constant which depends only on $N$.

Proof. Let $\phi: \mathbf{R} \rightarrow \mathbf{R}$ be an even $C^{\infty}$-function such that $\phi(0)=1$ and supp $\phi$ $\subset[-1,1]$. We consider $S \in \mathscr{R}$ such that

$$
\{S(\lambda)\}_{\alpha, \beta}= \begin{cases}\phi((2|\alpha|+n)|\lambda|) & \text { if } \alpha=\beta, \\ 0 & \text { otherwise, }\end{cases}
$$

for every $\lambda \in \mathbf{R}^{*}$. Then $S(\lambda)$ is the Fourier transform of a function in $S\left(H_{n}\right)$ (see [4], Theorem 1$)$. Obviously $G \in M(1, X)$. We consider

$$
K=\sum_{j=1}^{N}(-1)^{N+j}\left(\begin{array}{c}
N \\
j
\end{array}\right) G_{(j / h)}
$$

and $g_{h}=(-1)^{N+1} f * K$. We observe that $g_{h} \in M(h, X)$. Moreover

$$
f * K(u)=\int_{H_{n}} G(v) \sum_{j=1}^{N}\left(\begin{array}{c}
N \\
j
\end{array}\right) f\left(u \cdot \delta_{j / h} v^{-1}\right) d v .
$$

Since $\lim _{\lambda \rightarrow 0}\{\hat{G}(\lambda)\}_{\alpha, \alpha}=1$ for all $\alpha$, by (13) and the Lebesgue dominated convergence theorem we have $\int_{H_{n}} G=1$. Thus

$$
g_{h}(u)-f(u)=(-1)^{N+1} \int_{H_{n}} G(v) \Delta_{\delta_{1 / h} v}^{N} f(u) d v
$$

and

$$
\left\|g_{h}-f\right\| \leqslant \int_{H_{n}}|G(v)|\left\|\Delta_{\delta_{1 / h} v}^{N} f\right\| d v
$$


From Corollary 1 it follows that

$$
\begin{aligned}
\left\|g_{h}-f\right\| & \leqslant C^{\prime}(N) \omega_{N}(1 / h, f) \int_{H_{n}}|G(v)|(1+\rho(v))^{N} d v \\
& =C(N) \omega_{N}(1 / h, f) .
\end{aligned}
$$

THeORem 2. Let $h$ be a positive number and $D$ be a left-invariant differential operator with degree of homogeneity $N$. There exists a constant $C(D)$ such that $\|D f\| \leqslant C(D) h^{N}\|f\|$ for every $f \in M(h, X)$.

Proof. Let $\phi \in C_{c}^{\infty}([0,+\infty))$ such that $\phi(x)=1$ if $x \in[0,1]$. We define $S$ and $G$ as in Theorem 1. Since $f \in M(h, X)$ we have $f * G_{(1 / h)}=f$; hence $f$ is a $C^{\infty}$-function in $H_{n}$ such that (by (3) and (5))

$$
f *(D G)_{(1 / h)}=h^{-N} D\left(f * G_{(1 / h)}\right)=h^{-N} D f
$$

and

$$
\|D f\| \leqslant h^{N}\|D G\|_{1}\|f\|=C(D) h^{N}\|f\| \text {. }
$$

Remark. Suppose $f \in M(h, X)$; then $\hat{f}(\lambda)=0$ if $|\lambda|>h^{2} / n$ and for such $\lambda$ 's $D f(\lambda)=0$, for every invariant differential operator $D$. Therefore to avoid triviality we suppose $|\lambda| \leqslant h^{2} / n$. If $N$ is the degree of homogeneity of $D$, by (4) we have $\{D f(\lambda)\}_{\alpha, \beta}=0$ if $|\beta|>N+\left(k^{2} /|\lambda|-n\right) / 2$. Namely

$$
D f \in M(\sqrt{(2 N / n+1)}, X)
$$

(while in the abelian case we have $D f \in M(h, X)$ if $f \in M(h, X)$ ).

Suppose $n=1$. Let $\phi \in C_{c}^{\infty}\left(\mathbf{R}^{*}\right)$ and supp $\phi \subset\left(0, h^{2}\right]$. Let $S \in \mathscr{R}$ be such that

$$
\{S(\lambda)\}_{\alpha, \beta}= \begin{cases}\phi(\lambda) & \text { if } \alpha=\beta=0 \\ 0 & \text { otherwise. }\end{cases}
$$

Then $S=\hat{f}$ for some $f \in S\left(H_{1}\right)$ and $f \in M(h, X)$. By formula (4)

$$
\left\{\left(\bar{Z}^{N} f\right)^{\hat{n}}(\lambda)\right\}_{0, N}=\left((2|\lambda|)^{N} N !\right)^{1 / 2}\{\hat{f}(\lambda)\}_{0,0} .
$$

By the Plancherel formula we can choose a sequence of functions $\phi_{k}$ such that

$$
\left\|f_{k}\right\|_{2}=1 \text { and }\left\|\bar{Z}^{N} f_{k}\right\|_{2} \rightarrow\left(N ! 2^{N}\right)^{1 / 2} h^{N} \quad \text { as } k \rightarrow \infty \text {. }
$$

Hence the constant $C(D)$ of Theorem 2 is not necessarily equal to 1 . This contrasts with the abelian case, and with the situation for compact Lie groups (see [1], Lemma 2). 


\section{Lipschitz spaces}

We can apply the results of the previous sections to obtain a characterization of the Lipschitz spaces $\Lambda_{X}^{r}\left(H_{n}\right)$ by the behavior of their best approximation by functions of the classes $M(h, X)$.

Definition 3. Suppose $r>0$ and $N=[r]+1$. We say that the function $f$ belongs to the Lipschitz space $\Lambda_{X}^{r}\left(H_{n}\right)$ if $f \in X$ and there exists a constant $M=M(f)$ such that

$$
\left\|\Delta_{u}^{N} f\right\| \leqslant M \rho(u)^{r}
$$

for every $u \in H_{n}$.

The space $\Lambda_{X}^{r}$ becomes a Banach space if we put

$$
\|f\|_{\Lambda_{x}^{r}}=\|f\|+M_{f}
$$

where $M_{f}$ is the lower bound of all $M$ 's for which (16) is satisfied (compare with [3], Chapter 5-C).

THEOREM 3. The function $f$ belongs to $\Lambda_{X}^{r}\left(H_{n}\right)$ if and only if there exists $A>0$ and a family of functions $g_{h} \in M(h, X), h \geqslant 1$, such that

$$
\left\|f-g_{h}\right\| \leqslant A / h^{r} \text {. }
$$

Moreover, if $0 \leqslant k<r$ and $D \in V_{k}$, there exist two constant $C_{1}, C_{2}$ such that

$$
\begin{aligned}
\|D f\| & \leqslant C_{1}(\|f\|+A) \\
h^{k-r} \omega_{N}(h, D f) & \leqslant C_{2}(\|f\|+A) \quad \text { for every integer } N>r ;
\end{aligned}
$$

(obviously if $k=0$ we must replace $D f$ with $f$ in (18)).

Proof. We suppose $f \in \Lambda_{X}^{r}$. If we set $N=[r]+1$ in Theorem 1 , it follows by (14) and (15) that

$$
\begin{aligned}
\left\|f-g_{h}\right\| & \leqslant M \int_{H_{n}} \rho\left(\delta_{1 / h} v\right)^{r}|G(v)| d v \\
& =M h^{-r} \int_{H_{n}} \rho(v)^{r}|G(v)| d v=A h^{-r} .
\end{aligned}
$$

Vice versa we consider a sequence $g_{2^{j}} \in M\left(2^{j}, X\right)$ for which inequality (16) holds and we define

$$
Q_{0}=g_{1}, \quad Q_{j}=g_{2^{j}}-g_{2^{j-1}} \quad(j=1,2, \ldots) .
$$


Obviously $Q_{j} \in M\left(2^{j}, X\right)$ and by definition

$$
\begin{gathered}
\left\|Q_{0}\right\|=\left\|g_{1}\right\|<\|f\|+A, \\
\left\|Q_{j}\right\| \leqslant\left\|f-g_{2}\right\|+\left\|f-g_{2^{j-1}}\right\| \leqslant A\left(2^{r}+1\right) / 2^{r j}, \quad j=1,2, \ldots
\end{gathered}
$$

If $D \in V_{k}$, it follows from Theorem 2 that

$$
\begin{gathered}
\left\|D Q_{j}\right\| \leqslant C(D) 2^{k j}\left\|Q_{j}\right\| \leqslant C^{\prime} A 2^{(k-r) j}, \quad j=1,2, \ldots, \\
\left\|D Q_{0}\right\| \leqslant C^{\prime}(\|f\|+A) .
\end{gathered}
$$

In view of (20) $f=\sum_{j=0}^{\infty} Q_{j}$ in the sense of $X$. Moreover the estimates (21) show that the series $\sum_{j=0} D Q_{j}$ converges in $X$ to $D f$, if $D \in V_{k}(k<r)$. Hence $D f \in X$ and (17) holds.

We consider $u \in H_{n}$ and we choose a positive integer $K$ such that $2^{-(K+1)}<$ $\rho(u) \leqslant 2^{-K}$. If $N>r-k>0$ and $D \in V_{k}$ using inequalities (11) (with $\theta=0$ ) and (21) we obtain

$$
\left\|\Delta_{u}^{N} D Q_{j}\right\| \leqslant C(N) \rho(u)^{N} \sum_{D^{\prime} \in V_{N}}\left\|D^{\prime} D Q_{j}\right\| \leqslant C^{\prime} \rho(u)^{N} A 2^{j(N+k-r)} .
$$

Obviously

$$
\left\|\Delta_{u}^{N} D f\right\| \leqslant \sum_{j=0}^{K}\left\|\Delta_{u}^{N} D Q_{j}\right\|+\sum_{j=K+1}^{+\infty}\left\|\Delta_{u}^{N} D Q_{j}\right\|=J_{1}+J_{2}
$$

Now

$$
\begin{aligned}
J_{1} & \leqslant C^{\prime \prime}(\|f\|+A) \sum_{j=0}^{K} 2^{j(N+k-r)} \rho(u)^{N} \\
& \leqslant C^{\prime \prime \prime}(\|f\|+A) 2^{K(N+k-r)} \rho(u)^{N} \leqslant C^{\prime \prime \prime}(\|f\|+A) \rho(u)^{r-k}
\end{aligned}
$$

(the first inequality follows from (22) and the third one from the choice of $K$ ); moreover

$$
\begin{aligned}
J_{2} & \leqslant 2^{N} \sum_{j=M+1}^{+\infty}\left\|Q_{j}\right\| \leqslant A C^{\prime} \sum_{j=M+1}^{+\infty} 2^{j(k-r)} \\
& =A C^{\prime \prime} 2^{(M+1)(k-r)} \leqslant A C^{\prime \prime} \rho(u)^{r-k} .
\end{aligned}
$$

These estimates prove (18); if we set $k=0$, it follows that $f \in \Lambda_{X}^{r}$.

Remark. It follows from Theorem 3 that $f \in \Lambda_{X}^{r}$ implies $D f \in \Lambda_{X}^{s}$ where $s=r-k>0$ if $D \in V_{k}$. Now, let $k \geqslant 0$ and $N>r-k>0$. We can define on $\Lambda_{X}^{r}$ the following norms

$$
\begin{gathered}
\|f\|_{(N, k)}=\|f\|+\sup _{D \in V_{k}} \sup _{h>0} \omega_{N}(h, D f) / h^{r-k} \quad\left(\|f\|_{([r]+1,0)}=\|f\|_{A_{X}^{r}}\right), \\
\|f\|_{(*)}=\|f\|+\sup _{h>0} h^{r} \inf _{g_{h} \in M(h, X)}\left\|f-g_{h}\right\| .
\end{gathered}
$$


These norms are equivalent. In fact, let $M$ be an integer such that $M>r>k$ and $D \in V_{k}$. It follows from (14) and Lemma 3 that

$$
\begin{aligned}
\left\|f-g_{h}\right\| & \leqslant C^{\prime} h^{-k} \int_{H_{n}}|G(v)| \rho(v)^{k} \sum_{D \in V_{k}} \omega_{M-k}(\rho(v) / h, D f) d v \\
& \leqslant C^{\prime} h^{-k} \sup _{D \in V_{k}} \omega_{M-k}(1 / h, D f) \int_{H_{n}}|G(v)| \rho(v)^{k}(1+\rho(v))^{N-k} d v \\
& \leqslant C^{\prime \prime} h^{-k} \sup _{D \in V_{k}} \omega_{M-k}(1 / h, D f) .
\end{aligned}
$$

If we set $M-k=N>r-k$ we obtain

$$
h^{r} \inf _{g_{h} \in M(h, X)}\left\|f-g_{h}\right\| \leqslant C^{\prime \prime} h^{r-k} \sup _{D \in V_{k}} \omega_{N}(1 / h, D f) .
$$

Therefore

$$
\|f\|_{(*)} \leqslant C^{\prime \prime}\|f\|_{(N, k)}
$$

for every pair of integers $N$ and $k$ such that $k \geqslant 0, N>r-k>0$. On the other hand, it follows from (18) that

$$
h^{k-r} \omega_{N}(h, D f) \leqslant C^{\prime}\left(\|f\|+\sup _{\varepsilon>1} \varepsilon^{r} \inf _{g_{\varepsilon} \in M(\varepsilon, X)}\left\|f-g_{\varepsilon}\right\|\right) \leqslant C^{\prime}\|f\|_{(*)}
$$

\section{References}

[1] D. I. Cartwright and P. M. Soardi, 'Best conditions for the norm convergence of Fourier series', J. Approx. Theory 38 (1983), 344-353.

[2] J. Cygan, 'Subadditivity of homogeneous norms on certain nilpotent Lie group', Proc. Amer. Math. Soc. 83 (1981), 69-70.

[3] G. B. Folland and E. M. Stein, Hardy spaces on homogeneous groups (Mathematical Notes 28, Princeton Univ. Press, Princeton, N. J., 1982).

[4] D. Geller, 'Fourier analysis on the Heisenberg group', Proc. Nat. Acad. Sci. U.S.A. 74 (1977), 1328-1331.

[5] D. Geller, 'Local solvability and homogeneous distributions on the Heisenberg group', Comm. Partial Differential Equations 5 (1980), 475-560.

[6] I. R. Inglis, 'Bernstein's theorem and the Fourier algebra of the Heisenberg group', Boll. Un. Mat. It., to appear.

[7] P. M. Soardi, 'On non-isotropic Lipschitz spaces', Harmonic analysis (Lecture Notes in Math. 992, Springer-Verlag, Berlin, 1983).

\section{Dipartimento di Matematica}

Università di Milano

Via Saldini 50

20133 Milano

Italy 